\title{
Le reti alternative del cibo dopo la crisi. Teoria, ipotesi di lavoro e un caso studio esplorativo
}

Alternative food networks after the crisis. Theory, working hypotheses and an exploratory case study

\section{Giovanni Orlando}

\section{OpenEdition}

\section{Journals}

Edizione digitale

URL: http://journals.openedition.org/aam/324

DOI: $10.4000 /$ aam.324

ISSN: 2038-3215

\section{Editore}

Dipartimento Culture e Società - Università di Palermo

Notizia bibliografica digitale

Giovanni Orlando, «Le reti alternative del cibo dopo la crisi. Teoria, ipotesi di lavoro e un caso studio esplorativo ", Archivio antropologico mediterraneo [Online], Anno XXI, n. 20 (1) | 2018, online dal 30 juin 2018, consultato il 28 décembre 2019. URL : http://journals.openedition.org/aam/324 ; DOI : 10.4000/ aam.324

Questo documento è stato generato automaticamente il 28 dicembre 2019

Archivio antropologico mediterraneo 


\title{
Le reti alternative del cibo dopo la crisi. Teoria, ipotesi di lavoro e un caso studio esplorativo
}

Alternative food networks after the crisis. Theory, working hypotheses and an exploratory case study

\author{
Giovanni Orlando
}

\section{Introduzione}

1 Questo articolo si occupa delle dinamiche tramite cui i valori economici e culturali legati al cibo sono attualmente riprodotti e contestati nel sistema agro-alimentare - sia materialmente sia simbolicamente - come parte del sistema capitalistico nel suo complesso. Esso esplora questo tema specificamente in relazione ai cambiamenti che sono avvenuti nel mondo dell'economia solidale italiana dopo la crisi del 2008. Si possono ipotizzare quattro modalità con cui questo mondo è cambiato dopo la crisi e nel periodo di austerità: 1) generazione di nuovi fenomeni, 2) rafforzamento di quelli esistenti, 3) ostruzione e 4) irrilevanza. L'articolo analizza principalmente la prima possibilità, quella della generazione di nuovi fenomeni, prendendo in esame il caso della rete alternativa del cibo chiamata Fuori Mercato.

2 Negli ultimi trent'anni, le reti alternative del cibo (RAC) sono emerse dalle contraddizioni che hanno segnato la fine del secondo regime alimentare, quello industriale. Con il termine "regime alimentare/regime del cibo" (food regime) si indica il ruolo chiave che il cibo ha svolto dal XIX secolo in poi nel garantire la stabilità dell'accumulazione capitalistica su scala mondiale, ruolo che si è manifestato storicamente sotto forma di tre regimi specifici (Bernstein 2016; McMichael 2009).

Il primo regime, quello coloniale (circa 1870-1929), si è formato in conseguenza della rivoluzione industriale e della nascita delle classi operaie in Europa attraverso l'importazione di cereali, carne e prodotti tropicali come tè e caffè dalle colonie del Sud 
del mondo (Mintz 1986). Il secondo regime, quello industriale (circa 1945-1979), si basava sull'agricoltura intensiva del Nord al fine di esportare cereali (grano, riso, mais) a tariffe sovvenzionate verso le ex-colonie del Sud, consentendo così la sua parziale industrializzazione e assicurando nuovi mercati ai capitali euro-americani. Il regime attuale, quello corporate (1991-oggi), fa invece affidamento su una divisione globale del lavoro che obbliga i poveri del mondo a nutrire i ricchi sia nelle nazioni capitaliste più avanzate sia nelle economie emergenti del Sud, attraverso un sistema di commercio quello dei supermercati - che ha rivoluzionato l'approvvigionamento alimentare (McMichael, Friedmann 2007). In cambio delle calorie prodotte in massa che dal Nord raggiungono ancora i quattro angoli del pianeta, le regioni ricche ricevono quantità sempre maggiori di carne, pesce, frutta e verdura tramite filiere altamente specializzate che garantiscono il rifornimento degli scaffali 24 ore su 24,7 giorni su 7 . In questo regime, il capitale finanziario acquista un'importanza sempre maggiore, in parte grazie al ruolo svolto dalle catene commerciali multinazionali (Burch, Lawrence 2009).

4 Nel regime del cibo corporate si sono sviluppate due diverse modalità di approvvigionamento del cibo, che riflettono la tendenza della società mondiale a dividersi in classi transnazionali di consumatori ricchi e poveri. Forse gli esempi più evidenti di questa tendenza sono rappresentati da Walmart e Whole Foods, che si copiano a vicenda le linee di prodotti e le strategie dei prezzi pur definendo, almeno formalmente, due mercati per classi contrapposte (Friedmann 2005).

5 Per quanto i regimi del cibo garantiscano periodi stabili di accumulazione capitalista, le tensioni fanno sempre parte del sistema. I periodi di transizione sono importanti tanto quanto quelli di stabilità perché le regole che consentono l'estrazione di valore sono intrinsecamente sociali - il valore economico è un valore culturale, dopotutto; di conseguenza, l'accettazione o la contestazione delle "regole del gioco" rappresenta un fattore chiave di (in)stabilità. Due ambiti sono caratteristici delle contestazioni verso il regime corporate: la sovranità alimentare e la sostenibilità ambientale (Friedmann 1993; McMichael 2008). Dagli anni '70 in poi, i movimenti sociali hanno assunto un'importanza fondamentale nella definizione di questi due ambiti, importanza che è aumentata durante l'epoca della globalizzazione e del Washington consensus.

Da una parte, dunque, il regime attuale si fonda sui cosiddetti "imperi del cibo" (van der Ploeg 2009) che sono stati costruiti spingendo un modello di economia maggiormente disembedded - scorporata dalla società (Polanyi 2010 [1944]) - in cui la terra e il lavoro necessari per portare il cibo dal campo alla tavola sono sempre più soggetti all'autoregolazione dei mercati. Dall'altra, agricoltori, imprenditori, politici e comuni cittadini hanno unito le forze per contrastare questa mercificazione - che prende la forma di costi più elevati e prezzi più bassi per gli agricoltori (Heatherington 2011), allarmi per la sicurezza e le condizioni sanitarie degli alimenti per i consumatori, e un ambiente inquinato praticamente per tutti (Sage 2012) - e creare reti alternative del cibo ispirate a valori diversi da quelli del mercato.

Queste reti sono più diffuse nelle società benestanti del Nord, sebbene se ne possano trovare esempi anche nel Sud (Gregson, Ferdous 2014) e nel mondo post-socialista (Jung et al. 2014), a testimonianza di una storia che si è diffusa a livello globale: coltivare e consumare il cibo come sono stati abituati a farlo i paesi industrializzati dalla Seconda Guerra Mondiale in poi non è più possibile. Le RAC comprendono una grande varietà di fenomeni: dai mercati contadini ai gruppi d'acquisto solidale, dagli spacci delle aziende 
agricole al commercio equo e solidale, dall'agricoltura supportata dalle comunità alle aziende del web che consegnano cibo locale a domicilio e molto altro ancora (Goodman et al. 2012). I confini empirici e teorici di questi fenomeni con il regime corporate sono costantemente in divenire e ciò indica la presenza di una considerevole tensione all'interno del sistema.

8 A livello generale, le RAC si possono considerare un tentativo di far sì che la creazione di valore (Graeber 2001) nel sistema cibo avvenga con maggiore protezione sociale e ambientale rispetto allo stato attuale, soprattutto in un'economia che si sta rapidamente disembedding, scorporando dalla società (Pratt, Luetchford 2014). Storicamente, il processo di scorporamento dei mercati agroalimentari è stato causato da tre fattori che influenzano la creazione di valore: la distanza sociale tra agricoltori e consumatori, la durata degli alimenti prodotti industrialmente, e la costruzione culturale del cibo come merce (Friedmann 1994; Goody 1982)1. Favorendo scambi con un numero minore d'intermediari, dalle transazioni face to face a quelle spazialmente estese, le RAC cercano di reincorporare il cibo all'interno della società (Friedmann, McNair 2008). Promuovendo quelli che ritengono essere metodi di produzione meno intensivi (come quello biologico), esse cercano di reinserire il cibo nella natura (Kneafsey et al. 2008). Infine, svelando le conseguenze negative dell'ossessione del mondo moderno per i prezzi bassi e la convenienza, queste reti tentano di modificare la rappresentazione del cibo come merce (Petrini 2005).

Diversi tipi di RAC affrontano ciascuno dei tre fattori di creazione del valore in modi differenti (Marsden et al. 2000). Nella pratica, a mio avviso col tempo sono emerse due tendenze generali, basate sull'importanza relativa attribuita a due elementi: il tipo di prodotto venduto e il sistema di commercio impiegato. Alcune realtà si concentrano sulla diffusione di specifici prodotti "di qualità" a prescindere dal sistema di approvvigionamento, comprendendo perciò quelli convenzionali, dai supermercati ad Amazon (Fonte 2006); altre realtà, invece, cercano di costruire sistemi di approvvigionamento etici per i prodotti alimentari più in generale (Luetchford, Pratt 2011). Questa differenza empirica contribuisce a, e al contempo origina da, uno slittamento semantico nella nozione di "rete alternativa del cibo", dietro cui si celano due possibili interpretazioni: una debole (reti del cibo alternativo), e una forte (reti alternative del cibo) (Watts et al. 2005) ${ }^{2}$. Le due interpretazioni sono di per sé frutto di una tensione che trascende tutti i tipi di RAC, e più in generale, tutti i tipi di attività economica: la tensione fra mercato e società.

Sebbene l'Occidente si sia abituato a considerare "il mercato" come un'entità astratta la cui esistenza si fa via via più virtuale, i mercati rimangono delle istituzioni sociali costruite da élite di potere in condizioni storicamente specifiche, sulla base di interessi, norme e presupposti sulla proprietà e la moralità che nel corso del tempo sono suscettibili di cambiamento, rendendoli intrinsecamente arbitrari (Graeber 2012; Hann, Hart 2011). I mercati capitalisti sono speciali perché stabiliscono i prezzi in un modo apparentemente impersonale, attraverso il meccanismo della domanda e dell'offerta, e si basano sul denaro generalista (general-purpose money; cfr. Bohannan, Dalton 1962) per lo scambio di ogni sorta di beni, compresi i prodotti alimentari ma anche, e soprattutto, il lavoro umano. Per loro stessa natura, quindi, questi mercati possono trovare applicazione in ogni ambito del sociale e presso intere società che prima non li adottavano, potenziale che è stato enormemente amplificato dalla rivoluzione digitale. 
Qui risiede la tensione tra mercato e società, o la tensione nella crescita dell'uno a spese dell'altra (Gudeman 2008).

11 Considerando questa tensione dal punto di vista del valore e del modo in cui esso è creato, scambiato e utilizzato nel regime del cibo corporate, le RAC cercano d'indirizzare il flusso delle risorse naturali, del denaro, e dei significati culturali lontano dai mercati globali, dalle élite e dagli azionisti, verso i lavoratori, i cittadini e i territori. In altre parole, esse tentano di creare economie più protette - o chiuse - in cui sia possibile mantenere un certo grado di autonomia rispetto all'immenso potere esercitato dall'economia aperta (Luetchford 2014; Pratt 2014). Nell'epoca della Brexit e di Donald Trump, la metafora della "chiusura" può destare dubbi circa la natura politica (in senso ampio) dei fenomeni in questione (Orlando 2017). Il modo in cui Pratt e Luetchford usano tale metafora è però del tutto diverso. Per i due autori si tratta sostanzialmente di adattare al caso delle economie del cibo il concetto di "auto-protezione della società" di cui parlava Polanyi (2010 [1944]). Da questo punto di vista, quella di economia "chiusa" è un'utile metafora per cogliere determinati processi empirici e le loro fondamenta culturali, considerando che non sarà mai possibile - né auspicabile arrivare effettivamente a una chiusura totale.

Costruire un'economia agro-alimentare più chiusa significa rafforzare le relazioni sociali di collaborazione (o indebolire quelle di sfruttamento) tra mondo rurale e mondo urbano; significa garantire un prezzo giusto agli agricoltori e spingere affinché le retribuzioni di quelle classi di consumatori che non possono permettersi di pagare prezzi giusti siano aumentate; significa infine favorire regole sociali e ambientali più stringenti contro la deregulation normativa che caratterizza i mercati neoliberisti (De Neve et al. 2008). In questo modo, le RAC cercano di promuovere la trasformazione dell'economia basata sul "cibo dal nulla" in un'economia basata sul "cibo dei luoghi" (McMichael 2002).

\section{Le RAC dopo la crisi: ipotesi di lavoro}

Se, adattando il concetto di Carole Counihan (2004) del cibo come "voce" della modernità, è possibile affermare che le RAC rappresentano una voce dello scontro fra mercato e società, allora ha senso chiedersi quali effetti abbia prodotto su di esse la crisi economica iniziata nel 2008. Si tratta di una domanda cui è difficile dare risposta a causa della complessità del fenomeno e del fatto che quanto è accaduto in una sfera virtuale di scambio globale (il mercato dei mutui subprime) ha finito per avere ripercussioni assai diverse in giro per il mondo (Bear 2015; Hart 2012; Hsing 2012; Loftsdóttir 2014; Ottone 2012). In Europa, il diffondersi dell'austerità neoliberista come ideologia politico-economica dominante ne è stata la conseguenza principale (Varoufakis 2016). Ma anche l'austerità ha comportato esiti assai diversi all'interno del continente. In alcuni Paesi, per lo più dell'Europa meridionale o delle periferie di quella settentrionale, come l'Irlanda, al crac finanziario è seguita una crisi del debito sovrano che ha comportato dolorosi programmi di aggiustamento strutturale (Knight, Stewart 2016). Ciò non è avvenuto altrove. L'Italia, ad esempio, zoppica verso la stagnazione secolare (Carlini 2015; Furini 2012; Pipyrou 2014; Sassatelli et al. 2014), mentre Paesi come il Regno Unito e la Germania si sono ripresi molto più rapidamente, almeno in termini di PIL e tassi di occupazione. Naturalmente, l'austerità non riguarda soltanto il PIL e l'occupazione, ma anche i livelli di reddito e di disuguaglianza, gli orari lavorativi, 
l'assistenza sociale, quella sanitaria, e altro ancora. La frase "dopo la crisi", quindi, può essere letta in molti modi, ma principalmente in due; questa duplice interpretazione è possibile perché la "crisi" è allo stesso tempo un evento e un processo. Come evento, la frase "dopo la crisi" presuppone che questa sia finita; come processo, essa ammette la possibilità che la crisi sia ancora in corso - al di là degli indicatori macroeconomici poiché l'evento ha scatenato dei fenomeni di lunga durata dentro (durante) i quali le persone si trovano tuttora a operare.

Come processo, la crisi è figlia di «un prolungato esperimento sociale nel quale si è consentito a mercati e denaro di trovare la loro strada per il mondo» senza opporvi alcuna resistenza (Hart et al. 2010: 1). Il regime del cibo corporate è emerso durante questo "esperimento", noto col nome di globalizzazione neoliberista. Nel 2008 si è verificata una serie di eventi - dal collasso della banca d'investimenti Lehman Brothers ai salvataggi statali dei sistemi finanziari - che ha messo in moto nuovi processi, tra cui l'austerità, di cui molti Paesi devono ancora vedere la fine. Tenendo conto sia della dimensione evento sia di quella processo, allora, è possibile affermare di avere assistito a un'enorme riconfigurazione dei rapporti tra Stati, mercati e persone, in parte involontaria (come in un esperimento "naturale", cfr. Diamond, Robinson 2011), in parte frutto di precisi disegni politici. Questa riconfigurazione ha interessato le tre dimensioni tramite cui l'economia è incorporata nelle istituzioni sociali, ossia la reciprocità simmetrica, la redistribuzione centrale, e lo scambio di mercato (Harvey et al. 2007; Narotzky, Besnier 2014; Polanyi 1978 [1957]).

Le RAC cercano di regolare l'economia agro-alimentare soprattutto mediante una combinazione di reciprocità e scambio commerciale, pur beneficiando talvolta della redistribuzione centrale. Si possono quindi ipotizzare quattro modalità con cui questi fenomeni sono cambiati dopo la crisi e nel periodo di austerità. Chiamerò queste possibilità: generazione, rafforzamento, ostruzione e irrilevanza. Esse non sono mutualmente esclusive, verificandosi simultaneamente nel variegato mondo delle RAC, spesso persino nell'ambito della stessa iniziativa.

Da una parte, la crisi e l'austerità possono spianare la strada alla riscoperta dell'economia come progetto morale basato su valori non strumentali di mutualismo e cura per l'ambiente, portando così alla generazione e al rafforzamento ${ }^{3}$. La prima possibilità, dunque, è che nuove RAC siano state generate in seguito agli eventi degli ultimi dieci anni (Conill et al. 2012; Dickinson 2013; Rakopoulos 2014; Vournelis 2013), mentre la seconda è che RAC già esistenti si siano consolidate (Bramall 2011; Sonnino, Griggs-Trevarthen 2013; Guidi, Andretta 2015). L'elemento principale che distingue queste due possibilità è il tempo (in relazione allo spazio). Il tempo è un fattore importante perché vi è una considerevole differenza analitica tra iniziative che sono appena emerse, in Paesi come la Grecia o la Spagna (Calvário, Kallis 2016) e altre che esistono da almeno vent'anni, come l'economia solidale in Italia (Grasseni 2013). Considerati insieme, la generazione e il rafforzamento sembrano indicare una stratificazione di nuove iniziative su quelle più vecchie, fenomeno che potrebbe dipendere dall'alternarsi (nel tempo e nello spazio) di fasi di accelerazione e decelerazione degli effetti negativi dei mercati sulla società (Kalb 2012).

D'altra parte, il peggioramento delle condizioni di vita che è avvenuto nella maggior parte dei Paesi europei dopo il 2008, in particolare in quelli meridionali, potrebbe costituire un pericolo per le RAC sia in termini di ridimensionamento che di attrattiva. 
Ciò crea i presupposti per lo scenario dell'ostruzione (Brunori, Guarino 2010; GraciaArnaiz 2015).

18 Le RAC contano su varie forme di reciprocità per modificare gli scambi di mercato. Tuttavia, gli scambi che si svolgono al loro interno fanno pur sempre affidamento sul mezzo del denaro e sulla determinazione di prezzi di mercato. Poiché nell'economia convenzionale (quella aperta) sono sempre disponibili scelte più convenienti, i confini delle RAC sono potenzialmente sempre in discussione. L'erosione del potere d'acquisto dei consumatori può mettere a repentaglio la volontà delle persone di pagare prezzi più elevati, grazie ai quali nelle RAC si concretizza la reciprocità verso i produttori (Bondy, Talwar 2011; Carrigan, De Pelsmacker 2009; Hughes 2012). Dal punto di vista culturale, questa situazione sfida (potremmo dire "de-moralizza") i discorsi sul "cittadinoconsumatore" nelle classi medie su cui si basano la maggior parte delle RAC (Collins 2011; Johnston, Baumann 2010). Ma l'austerità può anche nuocere ai tentativi di aumentare l'attrattività delle RAC che si affidano alle istituzioni pubbliche per ridurne i costi strumentali, per esempio sotto forma di orti comunali, appalti "verdi", food policy council, ecc. In un'epoca di duri tagli alla spesa pubblica, le possibilità di risolvere il problema dell'elitarismo facendo ricorso alla redistribuzione centrale (statale) in aggiunta alla reciprocità e agli scambi di mercato appaiono più incerte che mai.

quarta e ultima possibilità consiste nell'impatto scarso o nullo dei processi attivati dalla crisi sui gruppi sociali coinvolti nelle RAC. Ciò non significa che questi fenomeni non siano cambiati nell'ultimo decennio, ma che tali cambiamenti sono stati causati da altri fattori, probabilmente come risultato di percorsi endogeni di più antica data.

In questo articolo analizzerò una di queste quattro possibilità, quella della generazione, attraverso il caso studio di una nuova RAC italiana, Fuori Mercato.

21 Il testo è così strutturato: nel paragrafo che segue, il terzo, espongo la metodologia della ricerca da cui è tratto l'articolo. Nel quarto paragrafo introduco gli attori principali del campo tramite una vignetta etnografica, mentre nel quinto discuto come il caso preso in esame illustra l'ipotesi della generazione. Nel sesto paragrafo analizzo i valori che accomunano gli attori incontrati sul campo; di converso, nel settimo metto in luce la composizione sfaccettata delle persone che animano le reti in questione. Le conclusioni chiudono l'articolo.

\section{Metodologia}

L'articolo si basa su una ricerca sul campo svolta principalmente nel 2015 e su periodi di ricerca intermittenti dopo quella data, che continuano tuttora ${ }^{4}$. La ricerca ha preso inizio da un'esaustiva e approfondita rassegna della letteratura scientifica sull'oggetto di studio condotta sia tramite portali accademici (es. cataloghi d'ateneo, Elsevier, ecc.) sia tramite cataloghi commerciali (es. Feltrinelli, Amazon, ecc.). La rassegna ha consentito di mettere a fuoco i principali interessi teorici della comunità scientifica sul tema in questione. A seguito della rassegna si è svolto un dettagliato campionamento delle iniziative riconducibili ai fenomeni discussi sopra in Italia; tale campionamento si è svolto principalmente tramite ricerche su Internet. Dopo avere costruito un quadro generale, la ricerca si è concentrata sulla RAC Fuori Mercato, nata originariamente sul territorio lombardo, più precisamente nell'hinterland milanese. Lo studio di questo caso si è articolato inizialmente tramite la raccolta del maggior numero possibile di dati secondari (es. articoli, comunicati stampa, documentari, servizi televisivi e radiofonici, 
ecc.). In seguito si è svolta la fase di raccolta dei dati primari, che è consistita in osservazione partecipante delle attività della rete e in interviste qualitative in profondità con informatori-chiave. Tutte le interviste e gli incontri più rilevanti sono stati audio-registrati. Considerata la nascita recente di Fuori Mercato e la sua continua evoluzione, l'articolo si focalizza in particolar modo sulla storia della rete fino a oggi, e va quindi considerato come uno studio esplorativo.

\section{Un caso studio esplorativo: Fuori Mercato}

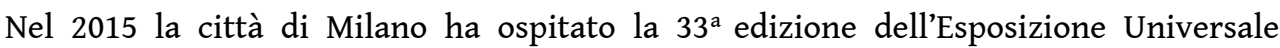
(Expo). Fin dalla sua prima edizione, tenutasi a Londra all'apice dell'epoca coloniale (nel 1851), l'Esposizione Universale ha accompagnato la storia del sistema-mondo moderno generando polemiche sempre maggiori (Harvey 1996; Sachs 2010). L'Expo di Milano era incentrata sul cibo $^{5}$. Obiettivo dell'evento, intitolato Nutrire il pianeta, Energia per la vita, era evidenziare "l'enorme sfida che rappresenta il raggiungimento di un equilibrio tra l'alimentazione dell'uomo e il rispetto per il pianeta" (Expo 2015). Elogiata da più parti, la manifestazione è stata nondimeno criticata per l'impatto sociale e ambientale negativo di molti dei suoi partner (Cospe 2015; Peracchi 2015) ${ }^{6}$. Il giorno successivo alla sua inaugurazione ero presente a un picnic di protesta contro l'evento organizzato da due reti di attivisti chiamate Genuino Clandestino e Fuori Mercato. Gli attivisti hanno parlato da un microfono in difesa dell'agricoltura contadina, accusando gli organizzatori dell'Expo di avere seguito la moda per arricchirsi col cibo. Il picnic si svolgeva - non a caso - davanti il punto vendita milanese di Eataly.

Eataly è una catena di vendita al dettaglio di prodotti alimentari di alta gamma sul modello dell'americana Whole Foods, che si è fatta un nome aggiungendo a quel modello raffinati ristoranti e spazi per corsi di cucina. Nella visione del suo fondatore, Oscar Farinetti (2009), chi visita Eataly può acquistare prodotti alimentari, ma anche mangiarli all'interno dei suoi punti vendita e conoscerli meglio attraverso eventi e percorsi formativi. Fin dalla nascita della catena, nel 2007, Eataly ha stabilito stretti rapporti con Slow Food grazie all'amicizia che lega Farinetti a Carlo Petrini, entrambi piemontesi (Bukowski 2015). Farinetti è stato anche un sostenitore dichiarato dell'Expo e questo, secondo i suoi critici, gli ha permesso di ottenere contratti redditizi per l'evento senza passare per le procedure di appalto pubblico (Ferrarella 2016). Eataly, già un simbolo di elitarismo gastronomico per diversi movimenti sociali che si oppongono alla globalizzazione neoliberista (Cfr. AA.VV, 2015), è finita così nel loro mirino per la presunta colpa di arruffianarsi i politici e le aziende che gestivano il megaevento. Per chi conosceva il passato dell'azienda, però, questa sintonia con il big business non è stata una sorpresa.

Prima di aprire Eataly, Farinetti era proprietario di Unieuro, la più grande catena italiana di elettronica di consumo (Sartorio 2008). All'inizio degli anni 2000 egli ha venduto la sua azienda al gruppo inglese Dixons Carphone per 230 milioni di sterline (Stevenson 2002), usando questi proventi per creare la sua nuova attività nel campo alimentare. Eataly sta ora per essere quotata in borsa, grazie a una forte presenza internazionale che include punti vendita a New York, Tokyo e Dubai (Insalaco 2017). Quest'operazione consentirà ad azionisti di tutto il mondo (potenzialmente anche a quelli del settore dell'elettronica) di re-investire capitali nel cibo di qualità. 

contraddistinguono il regime del cibo corporate delineato nell'introduzione dell'articolo. Le proteste contro di esso, e contro l'Expo, denunciavano proprio le caratteristiche di questo regime. Per riprendere la distinzione nella nozione di "rete alternativa del cibo" discussa nell'introduzione (quella tra un'interpretazione debole reti del cibo alternativo - e una forte - reti alternative del cibo), si può dire che Eataly è più vicina all'interpretazione debole, così come lo è il biologico venduto nei supermercati, mentre Genuino Clandestino e Fuori Mercato lo sono a quella più forte. Piero ${ }^{7}$, ad esempio, uno degli organizzatori della protesta, ha infatti descritto Farinetti con queste parole: "È uno molto furbo che cerca sempre di sussumerti; cerca di dirti che è dalla tua parte e poi ti neutralizza".

Furcato è un'associazione nata nel 2013 dalla collaborazione fra tre gruppi di persone: gli ex-lavoratori di una fabbrica del settore automotive di un piccolo Comune dell'hinterland milanese, chiusa nel 2012, un gruppo di piccoli produttori affiliati alla rete Genuino Clandestino, e alcuni consumatori che fanno parte di gruppi d'acquisto solidale (GAS) di Milano.

I lavoratori della fabbrica in questione sono coinvolti in un progetto per creare un'“impresa recuperata", espressione usata per indicare quelle aziende fallite che sono riavviate senza l'intervento dei precedenti proprietari (Bryer 2012, 2010). L'espressione viene dall'Argentina, dove la grave crisi economica verificatasi nel 2001 ha dato origine a numerosi casi di recupero. Gli operai milanesi non hanno riavviato la produzione di parti di automobili, che sarebbe risultata troppo costosa, ma hanno occupato il loro vecchio stabilimento, ormai vuoto, utilizzandolo per una serie di attività, una delle quali è Fuori Mercato ${ }^{8}$.

29 I piccoli produttori provengono dalla rete Genuino Clandestino e dal più vasto movimento del "post-biologico" (Moore 2006). Questa rete è stata creata nel 2009 nei pressi di Bologna da un gruppo di persone che intendeva denunciare il quadro normativo dell'Unione Europea che disciplina l'agricoltura biologica e i metodi di trasformazione degli alimenti, quadro che a loro avviso favorisce ingiustamente l'agroindustria'. L'iniziativa si è rapidamente trasformata in una campagna nazionale (Potito, Borghesi 2015). Pur concordando con i principi filosofici dell'agricoltura biologica - rispetto del suolo come organismo vivente e di tutta la biodiversità più in generale - questi coltivatori ritengono che l'attuale sistema europeo di certificazione e il settore della grande distribuzione che esso incoraggia non rappresentino quei principi e vadano pertanto superati. Poiché rifiutano le certificazioni ufficiali, il cibo che producono è per loro "genuino (e) clandestino". È in questo senso che la rete rappresenta una fase post-biologica del mondo delle RAC. Fuori Mercato comprende infine alcuni GAS che comprano il cibo direttamente da agricoltori biologici al fine di aggirare i supermercati (Tassan 2017).

\section{La crisi generatrice}

In che modo la crisi ha generato Fuori Mercato? La risposta risiede nell'attivazione di forme di mutualismo tra gli operai e coloro che li hanno aiutati a trovare nuove fonti di reddito dopo il licenziamento. È questa commistione di auto-aiuto e di aiuto dall'esterno (di solidarietà) ad aver dato origine a Fuori Mercato, come mostra 
chiaramente la storia dell'iniziativa, ricostruita di seguito sulla base delle interviste e delle fonti documentarie raccolte.

Quando gli operai decisero di occupare il loro stabilimento - all'inizio del 2013 - si resero subito conto che occorreva fare due cose: innanzitutto, trovare sostegno per la loro azione illegale tra la popolazione e le istituzioni del Comune di riferimento; poi utilizzare i capannoni per produrre nuovo reddito che consentisse loro di andare avanti. Essi hanno così intrapreso un notevole numero di iniziative, su cui non mi soffermerò in questa sede per ragioni di spazio. In quel periodo, uno dei loro contatti locali li ha presentati ad alcuni membri di un GAS (gasisti) di Milano, che hanno consigliato loro di preparare del cibo fatto in casa e di venderlo per raccogliere fondi in occasione dei tanti eventi che stavano organizzando. Oltre a ciò, i gasisti hanno consigliato agli operai di acquistare gli ingredienti nel vicino Parco Agricolo Sud Milano, una vasta area rurale protetta, il cui confine si trova a breve distanza dalla fabbrica occupata. Gli operai hanno così prodotto diverse centinaia di bottiglie di salsa di pomodoro e di limoncello.

I gasisti hanno anche suggerito di mettersi in contatto con un community shop di un paese vicino, dove i consumatori comprano prodotti locali direttamente dai coltivatori del Parco Agricolo. Nelle parole di Piero, membro della fabbrica recuperata: "C'è un GAS qui, che venivano a darci solidarietà, e c'hanno fatto capire che noi stavamo al confine del parco agricolo, proprio a 500 metri comincia il parco agricolo. Qui c'è un bel centro, che si chiama Buon Mercato, che già lavoravano insieme al GAS". Il negozio è gestito da un'associazione senza scopo di lucro e i suoi locali sono di proprietà del Comune; i clienti ordinano ciò che desiderano tramite un sito web e poi ritirano la merce di persona presso il negozio. I gestori hanno consigliato agli operai di organizzare un'attività analoga nella propria città, utilizzando il magazzino della fabbrica come punto di ritiro. Essi avrebbero condiviso il loro sito web e i suoi contatti, mentre gli operai avrebbero aiutato i gestori a raccogliere i prodotti presso gli agricoltori. Di nuovo Piero: "Buon Mercato ci ha chiesto se potevamo dare una mano per la distribuzione, e soprattutto per andare a fare la raccolta in giro. Dice: 'avete bisogno di un'attività, potreste fare il giro dei produttori e portare la roba a noi, e facciamo la distribuzione'. Anche perché c'era uno che lo faceva volontariamente, col suo camper, e dopo due anni s'ra un po' stufato". Anche in questo caso gli operai hanno messo in pratica il consiglio.

Fuori Mercato è sorta così, come un duplicato del community shop; anche il nome è un adattamento di quello (meno radicale) del negozio, chiamato Buon Mercato. L'intesa è andata avanti per alcuni mesi, fino a quando non è parso chiaro che la nuova iniziativa non attirava abbastanza clienti da risultare economicamente sostenibile ed è stata accantonata. Enzo, uno dei gasisti coinvolti, ha raccontato: "Mentre Buon Mercato sta in piedi, anche dando occupazione a tre part-time, loro non sono mai decollati, perché non sono mai andati oltre i 10-15 ordini alla settimana, un po' perché non si sono impegnati, un po' perché il territorio non era favorevole".

Nel frattempo, però, gli operai erano entrati in contatto con un altro gruppo, costituito da un'associazione di coltivatori biologici calabresi che aveva difficoltà a inviare piccole partite di cibo ai GAS milanesi per via dei costi in termini di tempo e denaro. Giacomo è stato l'operaio della fabbrica recuperata tramite il quale è avvenuto il primo contatto: "In una delle svariate serate in cui facevamo attività, si presentò un signore con un'amica, e parlando mi ha detto: 'Io conosco una realtà a Rosarno, ed eventualmente 
potrebbero essere interessati, loro producono agrumi, arance". Il problema di Rosarno era di organizzare meglio la distribuzione quassù a Milano. Dopo aver discusso il problema, i coltivatori si sono accordati con gli operai per usare il magazzino della fabbrica come struttura di stoccaggio, e hanno affidato loro la gestione degli ordini. In questo modo, i gruppi di famiglie di Milano, anziché ordinare individualmente direttamente all'associazione in Calabria, avrebbero inviato i loro ordini agli operai, $i$ quali li avrebbero raccolti, effettuando poi un unico ordine all'associazione. Quest'ultima avrebbe inviato tutta la spedizione al magazzino, dove gli operai avrebbero smistato la merce e consegnato le partite individuali alle singole famiglie. Questo accordo ha avuto molto più successo, se non altro perché i lavoratori non hanno dovuto crearsi una base di clienti dal nulla, ed esiste ormai da oltre quattro anni, rappresentando il fulcro del progetto di Fuori Mercato.

Vedendo il potenziale di questa forma di intermediazione, nel 2015 gli operai hanno cominciato a espandere l'attività, coinvolgendo coltivatori locali in grado di diversificare la gamma di prodotti offerta dai coltivatori calabresi. La formula è rimasta invariata, ma stavolta gli operai, in virtù delle distanze più brevi, possono ritirare $\mathrm{i}$ prodotti direttamente presso i coltivatori in Lombardia. In un certo senso, si tratta di una versione riveduta dello sfortunato progetto iniziale (il community shop), con la novità della consegna a domicilio. Per suscitare interesse nei confronti dell'iniziativa, gli operai si sono alleati con i membri milanesi di Genuino Clandestino e con altri attivisti locali, cominciando a organizzare dei mercati contadini durante i quali presentare la rete Fuori Mercato. Questi mercati si sono svolti in piazze pubbliche, ma anche in centri sociali occupati e all'interno della fabbrica recuperata, di solito durante eventi culturali. Oltre alla consegna a domicilio, gli operai offrono la possibilità di portare gli ordini a questi mercati, riducendo così il costo del servizio. Uno dei fautori di questo allargamento, Marco, ha descritto il processo così:

Siamo partiti ragionando sul fatto che questa stessa cosa [la collaborazione con Rosarno] potesse essere pensata anche per altre realtà, altri produttori, all'interno di un progetto che al momento cerca di coinvolgere una serie di realtà anche diverse, che siano spazi sociali occupati, GAS, associazioni varie ed eventuali, col tentativo di mettere al centro la questione del consumo critico, e di declinarlo nel senso: mettiamo insieme i produttori, i consumatori, in mezzo mettiamoci i mercatini contadini, mettiamoci le cucine popolari, ecc. ecc.

36 Il tentativo di espandere Fuori Mercato è stato riproposto a livello nazionale, ancora una volta con l'aiuto della rete Genuino Clandestino. Su questo fronte, gli operai hanno cercato di instaurare nuove collaborazioni con coltivatori affiliati alla rete in tutta la penisola italiana, soprattutto con quelli già coinvolti in progetti con particolari connotazioni etiche, come il soccorso ai lavoratori agricoli migranti o il recupero di varietà vegetali antiche. Nel 2015 la riunione annuale di Genuino Clandestino si è svolta nella fabbrica recuperata, dove sono stati consolidati i legami fra i due gruppi tramite la discussione degli interessi e delle problematiche comuni durante una serie di workshop e assemblee plenarie. È stato in questa occasione che si è ufficialmente discussa l'idea di un servizio di consegna nazionale.

\section{I valori alternativi del cibo}

Riflettere sul nome del progetto consente di cogliere il senso più generale del fenomeno Fuori Mercato. Particolarmente interessanti in tal senso sono le espressioni usate dai 
soggetti coinvolti per descrivere il problema dei supermercati e le sue possibili soluzioni. Le persone impegnate nella rete Fuori Mercato sottolineano costantemente l'importanza di "collegare" gli "anelli" e "chiudere" i "cerchi" e i "cicli" che rimangono aperti o interrotti a causa della grande distribuzione. Marco, ad esempio, ha detto: "Cerchi di creare un sistema integrato che dal produttore arriva al consumatore, ma che in realtà poi favorisca l'incontro, si superino i concetti di domanda e offerta classici". Enzo ha parlato di "una specie di chiusura del cerchio dell'economia solidale". Piero: "Comincia a costruire un giro del genere. Poi può essere il circolo ARCI, il CRAL, la mensa, il Comune. Puoi stabilire delle relazioni per cui crei un circuito alternativo". Giacomo, un altro ex-operaio, ha spiegato il progetto di Fuori Mercato in questo modo:

L'anello che vogliamo chiudere è quello della logistica. Per cui tu sei un bravissimo produttore, io sono un bravissimo consumatore, ma il trasporto lo dobbiamo sempre far fare a quelli là, la DHL, ecc. [...] Diventa un progetto che collega la penisola, che magari andrà da Mondeggi, la fattoria occupata, a prendere l'olio che faranno l'anno prossimo. Magari passerà da Caicocci, che è in Umbria, e prenderà le lenticchie e non lo so [cos'altro]. L'idea è quella di creare proprio una rete chiusa, che riesca ad alludere ad un sistema alternativo, completamente autonomo alla GDO [grande distribuzione organizzata].

Queste immagini trasmettono visivamente la nozione di un'economia chiusa che si tutela contro le sottrazioni di valore operate dall'economia aperta (quella capitalista). Grazie alla costruzione di un'economia chiusa di questo tipo, ci si procura il cibo "fuori mercato". La parola "mercato" è qui una sineddoche di "grande distribuzione", la complessità del primo ridotta alla specificità della seconda. È solo in questo senso ristretto che l'atto di evitare la grande distribuzione può rappresentare una demercificazione del cibo (mettere qualcosa fuori mercato significa smettere di venderla, dopotutto), anche se il cibo continua a essere scambiato attraverso il denaro a un prezzo stabilito (cioè continua a essere venduto). La classica definizione di merce è "un oggetto prodotto per essere venduto". Per i membri di Fuori Mercato, quindi, evitare la grande distribuzione significa trasformare il cibo-merce nel suo contrario, ossia in un bene comune.

I tre gruppi coinvolti - operai, agricoltori e consumatori - condividono quindi un atteggiamento critico nei confronti di quello che nell'introduzione è stato definito come il regime del cibo corporate, atteggiamento su cui si basa la loro collaborazione. In occasione di eventi pubblici, nei comunicati stampa e su Internet, Fuori Mercato ha fortemente criticato l'agricoltura intensiva, i supermercati, i fast food e le imprese agrochimiche. Il concetto di sovranità alimentare esercita una forte influenza sul progetto, soprattutto in seguito al coinvolgimento di Genuino Clandestino, che trae ispirazione dal movimento internazionale Via Campesina. Anche i contadini brasiliani senza terra, i Sem Terra, si sono rivelati un punto di contatto fra i vari operatori di Fuori Mercato. Dai Sem Terra, che fanno parte di Via Campesina, le fabbriche recuperate argentine hanno mutuato il motto ocupar, resistir, producir (occupa, resisti, produci), un motto che anche gli operai milanesi hanno fatto proprio, in parte per evocare i più famosi colleghi argentini e in parte per giustificare la loro collaborazione con gli agricoltori italiani. Nelle parole di Piero: "Abbiamo cominciato a conoscere il problema della sovranità alimentare, e le vecchie conoscenze del movimento dei Sem Terra, del movimento alter-mondialista, si sono combinate con le esperienze locali, che noi non conoscevamo perché eravamo metalmeccanici". Mentre Giacomo ha affermato: "Quello che stiamo facendo con Genuino Clandestino, con altre realtà che hanno scelto il loro 
impegno sull'autodeterminazione, la sovranità alimentare, ecc., con una serie d'incontri, con Joao Pedro Stedile dei Sem Terra, la vicinanza al Parco Agricolo, Expo, e tutto quello che ne deriva. L'idea è di ricominciare a parlare di cibo, perché tutti hanno bisogno di mangiare".

Una delle problematiche su cui i membri di Fuori Mercato focalizzano in particolar modo le loro critiche è il modo in cui forme di produzione e consumo del cibo come l'agricoltura biologica e il commercio equosolidale si sono oggi trasformate in possibilità di profitto per le grandi aziende. Le riflessioni di Marco in proposito sono particolarmente interessanti:

Ogni grande distribuzione ti fa la linea biologica. Non è più quella la battaglia. La battaglia è come faccio a scardinare tutte quelle che sono le dinamiche in essere che hanno portato ad esempio l'agricoltura a non produrre più per le necessità, ma per il mercato. Quindi il mio problema è pormi nell'ottica di dire 'come riesco a soppiantare in tutta la filiera, produzione, logistica e consumo, il sistema attuale'. Perché se uno solo di questi tre elementi lo lascio in mano alla grande distribuzione - al sistema - il resto diventa tutto assolutamente autoreferenziale. Riesci in qualche modo a fare una battaglia residuale. Ma poi il sistema sussume, perché il fatto che oggi tutti abbiano le linee biologiche significa che loro sono in grado di disinnescare l'aspetto rivoluzionario di un desiderio altro dal mercato. 'Mi piacerebbe avere cibo biologico...' Pronti, ce l'ho. E hai voglia tu a declinare il biologico diversamente da quello che fa l'Esselunga o la Coop. Allora quello che secondo me devi fare oggi è: tutto il progetto deve essere - come abbiamo appunto chiamato la nostra logistica - Fuori Mercato. Cioè uscire dalla logica di mercato.

Gli attivisti di Fuori Mercato ritengono che per impedire alle aziende di appropriarsi di altri settori alimentari, sia convenzionali che alternativi, sia fondamentale consolidare il rapporto tra piccoli produttori, che si ritiene coltivino in modo responsabile sotto il profilo ambientale e sociale, e chi intende (e può permettersi di) acquistare tali alimenti. Da questo punto di vista, consolidare significa principalmente organizzare l'approvvigionamento del cibo fuori dal settore della grande distribuzione.

Parlare di solidarietà verso lavoratori licenziati e della costruzione di un'economia più chiusa tra agricoltori e abitanti delle città può dare l'impressione di un processo coerente e a-problematico, caratterizzato da una politica lineare e da una progettualità che è limitata solo dalla volontà di chi vi è coinvolto. Nulla potrebbe essere più lontano dal vero. L'idea di cibo come nuovo bene comune si confronta con gli stessi problemi di concetti più vecchi, come quello di "comunità". Nella parte finale dell'articolo discuto quindi i fattori che tendono a separare i soggetti impegnati in questo progetto gli uni dagli altri.

\section{I limiti delle reti alternative del cibo}

Gli operai e i membri dei GAS che compongono Fuori Mercato sono consapevoli di essersi incontrati pur provenendo da contesti assai differenti. Ciò che ha spinto i primi a creare l'iniziativa è stata la ricerca di un nuovo lavoro, una ricerca iniziata quando la società che possedeva la loro fabbrica è entrata in amministrazione controllata, un anno dopo l'inizio della crisi finanziaria globale (nel 2009, ossia molto prima delle collaborazioni che ho descritto). Una volta che i clienti della fabbrica avevano rescisso i contratti con l'azienda, gli operai sono stati messi in cassa integrazione e gli amministratori hanno cominciato a cercare un nuovo proprietario. Questo periodo di incertezza è durato due anni, durante i quali gli operai hanno inscenato alcune 
manifestazioni di protesta per richiamare l'attenzione sui loro problemi e obbligare le autorità a trovare una soluzione. All'epoca, il loro obiettivo era trovare un nuovo acquirente e garantire nuovi investimenti per la fabbrica. Alla fine, un imprenditore polacco ha acquistato l'azienda approfittando dei generosi incentivi finanziari previsti dallo Stato italiano. Purtroppo, questa persona non era realmente interessata a investire nell'impresa, bensì ad acquisirne il nome, i brevetti e i macchinari e, una volta trascorso il termine obbligatorio di due anni per onorare l'accordo con le autorità, ha chiuso la fabbrica, licenziando tutti i dipendenti e trasferendo la produzione in Polonia. È a questo punto che alcuni operai hanno occupato lo stabilimento.

Di norma, le lotte dei lavoratori metalmeccanici per salvare il proprio posto di lavoro e le iniziative dei consumatori per aiutare i contadini o per proteggere la propria salute hanno poco in comune. Ma quando gli operai hanno occupato lo stabilimento, la prima idea che è venuta loro in mente è stata quella di riciclare materiali elettronici. Non è chiaro con quanta serietà essi abbiano assunto questo impegno, considerando la complessità del compito in termini di capitale, permessi e competenze. L'idea faceva probabilmente parte di una strategia per dare l'impressione di stare facendo "qualcosa di buono" dopo aver compiuto un'azione illecita di così alto profilo. La strategia si è rivelata vincente quando gli operai hanno ricevuto la solidarietà di alcuni gasisti, che hanno visto con favore l'intenzione di riconvertire lo stabilimento a una produzione sostenibile. Spesso i consumatori che ho conosciuto durante il campo hanno citato quest'impressione iniziale tra i motivi che li hanno spinti a prender parte al progetto, consapevoli che una "semplice" lotta per trovare nuovi posti di lavoro non avrebbe catturato la loro attenzione. Enzo, ad esempio, ha detto in proposito:

Se guardiamo alla loro condizione di partenza, dove comincia a connettersi? Che loro decidono che l'attività produttiva dovrà riprendere in chiave di riconversione ecologica. Non è che il GAS e il mondo dell'economia solidale non devono porsi il problema della salvaguardia dell'occupazione. Se lo devono porre in chiave di riconversione ecologica. Quindi per esempio quando ti dicono riconvertire le apparecchiature elettriche, vuol dire che si pongono il problema dei rifiuti, dell'utilizzo dell'energia, che comincia la sovrapposizione con le finalità del GAS. Diciamo un'attività di salvaguardia ambientale.

La collaborazione con i soggetti attivi sulla scena del cibo locale a Milano è stata tuttavia alquanto problematica. Come ho già discusso, la prima versione di Fuori Mercato (il clone del community shop) è fallita per mancanza d'interesse tra i clienti della provincia. Dopo quattro anni, l'attività principale del progetto (la vendita di prodotti alimentari nel milanese) raggiunge appena una ventina di GAS su un totale di quasi 200 presenti in zona. Gli operai e i consumatori che li aiutano lamentano il fatto che molti gruppi d'acquisto non sono interessati all'aspetto sociale del consumo di cibo locale, ma solo a quello salutistico, attribuendo a ciò la loro scarsa partecipazione e accusando di questo, in particolare, i GAS di più recente formazione. L'analisi di Piero è questa: "Le motivazioni sono le più varie. C'è chi l'ha fatto perché come mangi è una scelta politica. E c'è invece chi dice 'vabbè insomma', rispetto alle cose squallide che sei costretto a comprare al supermercato, 'voglio mangiare sano', e magari qualcuno ci aggiunge anche la difesa dell'ambiente. Però non è che m'interessa capire di più di questo. Se la Coop mi offre nel reparto biologico una cosa così, 'vabbè posso andare anche a prendermela, mi fido della Coop, che è una roba genuina', e quindi non compro le schifezze della Lidl". Tuttavia, anche tra i GAS che partecipano a Fuori Mercato è di norma solo un piccolo numero di membri a sollecitare la collaborazione, anziché il gruppo nel suo complesso. Stando a Enzo: "La scommessa è più sul futuro con loro - ma 
parlo di alcuni del GAS, di alcuni del DES [distretto di economia solidale], più che le varie realtà. Vale per alcuni dei vari gruppi, non vale per i gruppi in quanto tali questa esigenza".

Il problema sembra derivare dalla natura composita dell'economia solidale italiana, un fenomeno nato seguendo le forti connotazioni sociali che il concetto possiede in America Latina, che si è poi mescolato con l'incremento dei timori per la sicurezza alimentare avvenuto in Europa negli ultimi vent'anni. A peggiorare questa situazione, gli attivisti di Fuori Mercato sostengono che, da quando c'è stata la crisi, i consumatori etici sono diventati più attenti al prezzo $\mathrm{e}$, pertanto, non intendono sostenere neppure il piccolo costo extra dovuto per le consegne tramite Fuori Mercato. Giacomo ha detto in proposito: "Purtroppo molte volte i GAS sono gruppi d'acquisto, e non sono più solidali, ma vogliono risparmiare come vogliono risparmiare tutti, quando invece è anche e soprattutto il progetto che c'è dietro un produttore che determina l'eticità e il voler acquistare quel prodotto a differenza di un altro, che magari è un rispettabilissimo produttore biologico, che costa meno di quell'altro".

Quest'ultimo problema ne aggrava un altro: le divergenze sull'effettiva necessità (o almeno sull'opportunità) di quest'attività di consegna, visto che mettere in contatto coltivatori e abitanti delle città è comunque una forma d'intermediazione, cosa che rappresenta una sorta di anatema per quasi tutti nell'economia solidale italiana. Enzo ha espresso questo timore:

Se poi decolla il progetto... Certo che poi alcuni GAS lo vedrebbero male, o non bene, quello di dire a Terra e il cielo [un produttore biologico], che ti porta i prodotti una volta al mese, 'no, portali qua due volte l'anno, poi i GAS si riferiranno qua'. Qualche GAS sicuramente non la vedrà bene, perché qualche problema lo pone dal punto di vista dell'intermediazione. Si configurerebbe anche qui il rischio che diventi non solo un polo logistico ma un nuovo negozio.

In Italia, è stato il commercio equosolidale a influenzare le caratteristiche dell'economia solidale a partire dagli anni ' 90 , soprattutto l'idea di avere rapporti diretti con gli agricoltori in un contesto locale in cui era effettivamente possibile incontrarli di persona (rispetto a quelli dei paesi in via di sviluppo). Gli intermediari sono a lungo stati gli archetipi del "cattivo" per il commercio equo. Così, nonostante questa serie d'idee abbia prodotto molti effetti positivi, ha anche creato nei consumatori che partecipano alle RAC l'aspettativa che siano i coltivatori stessi a consegnare i loro prodotti in città. $\mathrm{E}$ mentre il costo della benzina è di solito incluso nel calcolo del prezzo, quello della consegna - in quanto lavoro - di solito non lo è. Inoltre, tutte le mansioni necessarie per far funzionare un GAS (ricevere gli ordini dalle famiglie, raggrupparli, inviarli all'agricoltore, e infine pagarlo) vengono svolte solitamente su base volontaria - gratuita - dagli stessi membri del gruppo. Secondo Giacomo: "Il problema è che purtroppo quello che è per la quasi totalità il servizio di gestione dei GAS è basato sul volontariato, che cozza con una gestione che deve essere quella di ordini, spedizioni, bancali, merce da controllare, ecc., che ha bisogno d'interventi precisi, puntuali e non può essere affidata al volontarismo".

I lavoratori e i consumatori di Fuori Mercato sono consci di questi problemi e hanno cercato di risolverli in tre modi: in primo luogo, facendo presente che loro non possono essere considerati intermediari nel senso dispregiativo del termine, applicato ai grossisti o alle società commerciali che si occupano di consegne. A loro avviso questo paragone sarebbe assurdo. In secondo luogo, essi sostengono che il servizio che offrono semplifica in misura considerevole sia la vita degli agricoltori sia quella dei 
consumatori, soprattutto in un'area fortemente urbanizzata come quella di Milano, risparmiando in tal modo energie che possono invece essere usate per la diffusione di queste iniziative. Come ha spiegato Enzo: "Perché questo rapporto della filiera corta a quel livello deve rimanere, delle visite ai produttori, della conoscenza. Questo può liberare risorse per i GAS, non dovendo più fare il lavoro di consegna come si fa adesso, possono buttare energie su aspetti più politici del lavoro dell'economia solidale. Però non è facile, perché non viene colta ancora questa esigenza". In terzo luogo, i due gruppi sono dell'idea che ogni tipo di lavoro debba essere equamente retribuito, compreso quello di portare il cibo direttamente dal campo alla tavola, con tutto ciò che questo comporta, dall'evasione delle pratiche alla guida del furgone. Giacomo: "In generale pensiamo che il lavoro svolto debba essere pagato. Dietro la gestione degli ordini, delle spedizioni, ecc., c'è un lavoro enorme". Queste osservazioni, benché valide, non hanno cambiato in maniera apprezzabile il rapporto con l'economia solidale locale. I tentativi di espandere la rete dei collaboratori e dei simpatizzanti che ho descritto in precedenza mirano precisamente a modificare questo stato di cose.

Anche il rapporto con gli agricoltori si è rivelato non sempre lineare. Per loro stessa ammissione, gli operai e i consumatori sanno che gli agricoltori contribuiscono solo in minima parte alle decisioni relative al progetto, soprattutto perché sono sparpagliati, talvolta a grande distanza da Milano, e sono già oberati di lavoro, avendo poco tempo e attitudine per partecipare a riunioni interminabili. Stando a Piero: "I produttori sono poco integrati in questa roba, di più sono il giro dei consumatori. Magari aumentando il numero dei produttori verrà dentro il loro punto di vista e ci aiuteranno anche a perfezionare alcune cose, dicendo 'guarda che non funziona come tu ti sei immaginato, perché io ho anche questo problema"”.

51 Ci sono però anche altre questioni, maggiormente attinenti al processo materiale $\mathrm{e}$ simbolico che consente di trasformare una merce in un bene comune, o mettere il cibo "fuori mercato". Qui il punto cruciale è costituito dal tentativo di evitare i rivenditori convenzionali per costruire un'economia più chiusa, in cui il valore resti nelle mani di chi lo crea. Gli agricoltori non si fanno particolari problemi se Fuori Mercato agisce da intermediario e consegna i loro prodotti alle famiglie (a differenza di quanto pensano alcuni consumatori, come appena discusso). Le cose cambiano però quando le loro intenzioni sono messe in discussione a causa dei rapporti che alcuni intrattengono con rivenditori commerciali.

Molte delle persone alla guida del progetto Fuori Mercato hanno convinzioni politiche abbastanza forti, il che li porta ad avere un atteggiamento che si può definire una sorta di "boicottaggio". Non si tratta di vere e proprie campagne di boicottaggio organizzato, ma piuttosto di una serie di decisioni che riguardano le persone con cui collaborare. Un esempio di questo atteggiamento è stata la decisione di non acquistare più i pomodori per le bottiglie di salsa destinate alle raccolte fondi da un coltivatore locale che aveva preso parte all'Expo di Milano. Un altro è stato il rifiuto di partecipare a una giornata di eventi, che includeva anche un mercato contadino, perché sponsorizzata dall'Expo.

53 Pur tutelando i principi etici, questa strategia può diventare problematica. Per gli agricoltori, la possibilità di porre il cibo "fuori mercato" è ottima purché ciò riguardi l'intera produzione dell'azienda, non solo una piccola parte. Se si avvera solo la seconda possibilità, essi devono trovare altre soluzioni per la loro sopravvivenza. Marco spiega molto bene la situazione: "Su quell'aspetto lì poi c'è la discussione, sapendo che io non ho una soluzione, perché alla fine del ragionamento giustamente uno dice 'benissimo, 
quando io ho prodotto 100 , e tu con tutto il tuo ragionamento me ne porti via 10 , gli altri 90 che faccio?"'.

Alcuni coltivatori locali che fanno parte di Fuori Mercato hanno rapporti commerciali anche con Eataly, soprattutto quelli specializzati in alimenti artigianali, fatto che ha portato ad altri attriti. Nel racconto di Marco:

Diciamo che ci siamo orientati rispetto ad alcune questioni di fondo: produzione biologica ma non necessariamente certificata, piccoli produttori, non rivenditori [di cibo altrui], e che non avessero connessione o con la grande distribuzione e soprattutto - questo è diventato poi uno degli elementi critici in questo periodo non avessero nessuna relazione con Expo. Allo stato attuale credo si debba rivedere un attimino questo disciplinare, perché in alcune situazioni abbiamo elementi critici. Noi abbiamo produttori a cui non si può dire assolutamente niente, che però ti dicono 'Eataly mi offre di tenere le mie farine, a me piacerebbe potergli dire di no, ma non sono nella condizione di farlo, perché devo tenere in piedi la mia attività'.

Stando a Marco, persino l'associazione di agricoltori calabresi, il maggior alleato di Fuori Mercato, vende buona parte delle sue arance biologiche a una piattaforma di distribuzione locale, tramite cui esse finiscono probabilmente sugli scaffali dei supermercati. Ancora Marco: "Io credo che tu non ti possa semplicemente limitare a dire allora tu sei cattivo che vai col nemico. No. Bisogna assumere questa come una problematica. Devi dire: 'Io movimento complessivo del consumo critico non sono in grado di assorbire tutta la produzione"”.

\section{Conclusioni}

I lavoratori e i consumatori di Fuori Mercato sono consapevoli delle tensioni e delle contraddizioni analizzate nel paragrafo precedente. Il loro è per lo più un atteggiamento pragmatico e, soprattutto, non è una posizione estremista fine a se stessa. Quando discutono di queste tensioni e contraddizioni, parlano spesso dello stato dei "rapporti di potere" o dei "rapporti di forza", ammettendo che possono fare poco per cambiarli. Queste espressioni vivide riportano al quadro più ampio di un regime del cibo corporate le cui forze centrifughe parcellizzano i luoghi della coltivazione, trasformazione, distribuzione e consumo. La finanziarizzazione è un fattore chiave di questo processo di apertura economica, che riflette il ruolo sempre maggiore svolto dalla finanza nel mondo capitalista. Il crac del 2008 è stato uno dei maggiori campanelli d'allarme di questo ruolo, e le sue conseguenze si possono ancora avvertire.

Traendo spunto da una lunga linea di pensiero antropologico (Graeber 2001, 2012; Gudeman 2008; Hann, Hart 2011; Hart et al. 2010; Narotzky, Besnier 2014; Polanyi 1978 [1957], 2010 [1944]), ho proposto di vedere l'austerità come una riconfigurazione pianificata dei confini tra mercato e società. Questi confini si sono spostati parecchie volte da quando è nato il capitalismo - allargandosi e restringendosi - e la tensione connaturata a questo processo affiora ovunque nel nostro mondo. Le reti alternative del cibo sono un esempio di come il principio della protezione sociale, ivi compresa la tutela della natura, si applichi nell'ambito di esperimenti concreti per contenere il principio di mercato. Di fronte ai rinnovati assalti di quest'ultimo, sembrano essere in gioco quattro possibilità: generazione, rafforzamento, ostruzione e irrilevanza. In questo articolo ho deciso di soffermarmi sulla creazione di nuove iniziative, senza passare sotto silenzio $i$ tanti ostacoli che intralciano la teoria, ma soprattutto la pratica, del cibo come un nuovo bene comune. 


\section{BIBLIOGRAFIA}

Aa.Vv., 2015 Expo 2015. Il cibo che affama, Luce Edizioni, Torino.

Bear L., 2015 Navigating Austerity: Currents of Debt along a South Asian River, Stanford University Press, Chicago.

Bernstein H., 2016 «Agrarian political economy and modern world capitalism: the contributions of food regime analysis», in The Journal of Peasant Studies, 43 (3): 611-647.

Bohannan P., Dalton G. (eds)

1962 Mercati d'Africa, Franco Angeli, Milano.

Bondy T., Talwar V., 2011 «Through thick and thin: how fair trade consumers have reacted to the global economic recession», in Journal of Business Ethics, 101: 365-383.

Bramall R., 2011 «Dig for victory! Anti-consumerism, austerity and new historical subjectivities», in Subjectivity: 4, 68-86.

Brunori G., Guarino A., 2010 «Security for whom? Changing discourses on food in Europe in times of a global food crisis», in G. Lawrence et al. (eds), Food Security, Nutrition and Sustainability, Earthscan, London: 41-60.

Bryer A., 2012 «The politics of the social economy: A case study of the Argentinean empresas recuperadas», in Dialectical Anthropology, 36: 21-49.

Bryer A., 2010 «Beyond bureaucracies? The struggle for social responsibility in the Argentine workers cooperatives», in Critique of Anthropology, 30 (1): 41-61.

Bukowski W., 2015 La danza delle mozzarelle. Slow food, Eataly, Coop e la loro narrazione, Edizioni Alegre, Roma.

Burch D., Lawrence G., 2009 «Towards a third food regime: behind the transformation», in Agriculture and Human Values, 26: 267-279.

Calvário R., Kallis G., 2016 «Alternative food economies and transformative politics in times of crisis: insights from the Basque country and Greece», in Antipode, 49 (3): 597-616.

Carlini R., 2015 Come siamo cambiati. Gli italiani e la crisi, Laterza, Roma-Bari.

Carrigan M., De Pelsmacker P., 2009 «Will ethical consumers sustain their values in the global credit crunch?», in International Marketing Review, 26 (6): 674-687.

Collins J., 2011 «Wal-Mart, American consumer citizenship, and the 2008 recession», in Focaal Journal of Global and Historical Anthropology, 61: 107-116.

Conill J. et al., 2012 «Beyond the crisis: the emergence of alternative economic practices», in M. Castells et al. (eds), Aftermath: The Cultures of the Economic Crisis, Oxford University Press, Oxford: 210-250.

Cospe, 2015 «L'inaccettabile kermesse dell'insostenibilità». http://www.cospe.org/news/cospeed-expo-2015-linaccettabile-kermesse-dellinsostenibilita/ (ultimo accesso 27/04/2015).

Counihan C., 2004 Around the Tuscan Table: Food, Family and Gender in Twentieth century Florence, Routledge, London.

Crouch C. et al., 2016 «Democracy in neoliberalism? », in Anthropological Theory, 16 (4): 497-512. 
Della Porta D., 2015 Social Movements in Times of Austerity: Bringing Capitalism back into Protest Analysis, Polity, Cambridge.

De Neve G. et al., 2008 «Introduction: revealing the hidden hands of global market exchange», in G. de Neve et al. (eds), Hidden Hands in the Market: Ethnographies of Fair Trade, Ethical Consumption and Corporate Social Responsibility, Emerald, Bingley: 1-30.

Diamond J., Robinson J., 2011 Esperimenti naturali di storia, Codice Edizioni, Torino.

Dickinson M., 2013 «Cooking up a revolution: food as a democratic tactic at Occupy Wall Street», in Food, Culture \& Society, 16 (3): 359-365.

Expo, 2015 «The meaning of Expo». http://www.expo2015.org/en/learn-more/the-history-/themeaning-of-expo (ultimo accesso 27/04/2015).

Farinetti O., 2009 Coccodè. Il marketing-pensiero di Oscar Farinetti, Giunti, Milano.

Ferrarella L., 2016 «Il giudice archivia Sala su Eataly: all'Expo non ci fu abuso d'ufficio». http:// milano.corriere.it/notizie/cronaca/16_gennaio_19/giudice-archivia-sala-eataly-all-expo-non-cifu-abuso-d-ufficio-c82abfec-be8d-11e5-8000-980215fcd4e6.shtml (ultimo accesso 06/11/17).

Fonte M., 2006 «Slow food's presidia: what do small producers do with big retailers?», in T. Marsden, Murdoch, J. (eds), Between the Local and the Global: Confronting Complexity in the Contemporary Agri-Food Sector, Research in Rural Sociology and Development 12, Elsevier, Oxford: 203-240.

Friedmann H., 2005 «From colonialism to green capitalism: social movements and emergence of food regimes», in F. H. Buttel, McMichael, P. (eds), New Directions in the Sociology of Global Development, Research in Rural Sociology and Development, 11, Elsevier, Oxford: 227-264.

Friedmann H., 1994 «Distance and durability: shaky foundations of the world food economy», in P. McMichael (ed.) The Global Restructuring of Agro-food Systems, Cornell University Press, Ithaca (NY).

Friedmann H., 1993 «After Midas's feast: alternative food regimes for the future», in P. Allen (ed.), Food for the Future: Conditions and Contradictions of Sustainability, Wiley, New York: 213-233.

Friedmann H., McNair A., 2008 «Whose rules rule? Contested projects to certify 'local production for distant consumers' «, in Journal of Agrarian Change, 8 (2-3): 408-34.

Furini L., 2012 L'Italia in bolletta. Risparmi in fumo, debiti alle stelle: come si estingue il ceto medio, Garzanti, Milano.

Goodman D. et al., 2012 Alternative Food Networks: Knowledge, Practice, and Politics, London, Routledge.

Goody J., 1982 Cooking, Cuisine and Class: A Study in Comparative Sociology, Cambridge University Press, Cambridge.

Gracia-Arnaiz M., 2015 «Eating in a time of 'crisis': new health and dietary contexts in Spain», in L. Escajedo San-Epifanio, De Renobales Scheifler M. (eds), Envisioning a Future without Food Waste and Food Poverty: Societal Challenges, Wagenigen, Wagenigen Academic Publishers: 219-224.

Graeber D., 2012 Debito. I primi 5000 anni, il Saggiatore, Milano.

Graeber D., 2001 Toward an Anthropological Theory of Value: The false Coin of Our Own Dreams, Palgrave Macmillan, New York.

Grasseni C., 2013 Beyond Alternative Food Networks: Italy's Solidarity Purchase Groups, Bloomsbury, London. 
Gregson N., Ferdous R., 2014 «Making space for ethical consumption in the South», in Geoforum, 67: 244-255.

Gudeman S., 2008 Economy's Tension: The Dialectics of Community and Market, Berghahn, Oxford.

Guidi R., Andretta M., 2015 «Between resistance and resilience: how do Italian solidarity purchase groups change in times of crisis and austerity?», in Partecipazione e Conflitto, 8 (2): 443-447.

Hann C., Hart K., 2011 Antropologia economica. Storia, etnografia, critica, Einaudi, Torino.

Hart K., 2012 «The roots of the global economic crisis», in Anthropology Today, 28 (2): 1-3.

Hart K. et al., 2010 «Building the human economy together», in K. Hart et al. (eds), The Human Economy: A Citizen Guide, Cambridge, Polity: 1-17.

Harvey M. R. et al., 2007 Karl Polanyi: New Perspectives on the Place of the Economy in Society, Manchester University Press, Manchester.

Harvey P., 1996 Hybrids of Modernity. Anthropology, the Nation State and the Universal Exhibition, Routledge, London.

Heatherington T., 2011 «Introduction: remaking rural landscapes in twenty-first century Europe», in Politicking the Farm: Transitions and Transformations in European Agriculture, Anthropological Journal of European Cultures, 20 (1): 1-9.

Hsing Y., 2012 «No crisis in China? The rise of China's social crisis», in M. Castells et al. (eds), Aftermath: The Cultures of the Economic Crisis, Oxford University Press, Oxford: 251-277.

Hughes A., 2012 «Corporate ethical trading in an economic downturn: recessionary pressures and refracted responsibilities», in Journal of Economic Geography, 12: 33-54.

Insalaco C., 2017 «Eataly, il regalo per i dieci anni è la Borsa». http://www.lastampa.it/ 2017/01/27/cronaca/la-festa-da-eataly-il-regalo-per-east-diecianni-la-borsa-farinetti-adessopuntiamo-a-cina-india-e-far-1VU7UN4zoA7aG9v5htTlHO/pagina.html (ultimo accesso 13/03/17).

Johnston J., Baumann S., 2010 Foodies: Democracy and Distinction in the Gourmet Foodscape, Routledge, London.

Jung, Y. et al. (eds), 2014 Ethical Eating in the Postsocialist and Socialist World, University of California Press, Berkeley.

Kalb D., 2012 «Thinking about neoliberalism as if the crisis was actually happening», in Social Anthropology, 20 (3): 318-330.

Kneafsey M. et al., 2008 Reconnecting Consumers, Producers and Food. Exploring Alternatives, New York, Berg.

Knight D., Stewart C., 2016 «Ethnographies of austerity: temporality, crisis and affect in southern Europe», in History and Anthropology, 27 (1): 1-18.

Lekakis E. J., 2017 «Economic nationalism and the cultural politics of consumption under austerity: The rise of ethnocentric consumption in Greece», in Journal of Consumer Culture, 17 (2): 286-302.

Loftsdóttir K., 2014 «Iceland, rejected by McDonald's: desire and anxieties in a global crisis», in Social Anthropology, 22 (3): 340-353.

Luetchford P., 2014 «Food and consumption», in J. Pratt, Luetchford P. (eds), Food for Change: The Politics and Values of Social Movements, Pluto Press, New York: 47-70. 
Luetchford P., Pratt J., 2011 «Values and markets: an analysis of organic farming initiatives in Andalusia», in Journal of Agrarian Change, 11 (1): 87-103.

Marsden T. et al., 2000 «Food supply chain approaches: exploring their role in rural development», in Sociologia Ruralis, 40 (4): 424-438.

McMichael P., 2009 «A food regime genealogy», in The Journal of Peasant Studies, 36 (1): 139-169.

McMichael P., 2008 «Peasants make their own history, but not just as they please...», in Journal of Agrarian Change, 8 (2-3): 205-228.

McMichael P., 2002 «La restructuration globale des systems agro-alimentaires», in Mondes en Developpment, 30 (117): 45-54.

McMichael P., Friedmann H.

2007 «Situating the 'retailing revolution' «, in D. Burch, Lawrence, G. (eds), Supermarkets and Agrofood Supply Chains. Transformations in the Production and Consumption of Foods, Edward Elgar, Cheltenham.

Mintz S., 1986 Sweetness and Power. The Place of Sugar in Modern History, Vintage, New York.

Moore 0., 2006 «Understanding postorganic fresh fruit and vegetable consumers at participatory farmers' markets in Ireland: reflexivity, trust and social movements», in International Journal of Consumer Studies, 30 (5): 416-426.

Narotzky S., 2016 «Between inequality and injustice: dignity as a motive for mobilization during the crisis», in History and Anthropology, 27 (1): 74-92.

Narotzky S., Besnier N. (eds), 2014 «Crisis, value, and hope: rethinking the economy», Supplement 9, Current Anthropology, 55: 1-154.

Orlando G., 2017 «Solidarity economies in the age of Brexit and Trump», Palaver 6 n.s., n.2, 165-178.

Ottone E., 2012 «A non-global crisis? Challenging the crisis in Latin America», in M. Castells et al. (eds), Aftermath: The Cultures of the Economic Crisis, Oxford, Oxford University Press: 278-302.

Peracchi E., 2015 «Il Forum Diritti Lavoro denuncia: "Volontari irregolari, Expo risparmia milioni"». http://video.repubblica.it/edizione/milano/milano-il-forum-diritti-lavoro-denunciavolontari-irregolari-expo-risparmia-milioni/197481/196509?ref=search (ultimo accesso 27/04/2015).

Petrini C., 2005 Buono, pulito e giusto: principi di nuova gastronomia, Einaudi, Torino.

Ploeg, van der J. D., 2009 I nuovi contadini. Le campagne e le risposte alla globalizzazione, Donzelli, Roma.

Polanyi K., 2010 [1944], La grande trasformazione: le origini economiche e politiche della nostra epoca, Torino, Einaudi.

1978 [1957] «L'economia come processo istituzionale», in Polanyi et al. (a cura di), Traffici e mercati negli antichi imperi, Einaudi, Torino: 315-326.

Potito M., Borghesi R., 2015 Genuino clandestino. Viaggio tra le agri-culture resistenti ai tempi delle grandi opere, Terra Nuova Edizioni, Firenze.

Pratt J., 2014 «Farming and its values», in J. Pratt, Luetchford P. (eds), Food for Change: The Politics and Values of Social Movements, Pluto Press, New York: 24-46.

Pratt J., Luetchford P. (eds), 2014 «Introduction», in Food for Change: The Politics and Values of Social Movements, Pluto Press, New York: 1-23. 
Pipyrou S., 2014 «Cutting bella figura: irony, crisis, and secondhand clothes in South Italy», in American Ethnologist, 41 (3): 532-546.

Rakopoulos T., 2014 «The crisis seen from below, within, and against: from solidarity economy to food distribution cooperatives in Greece», in Dialectical Anthropology, 38: 189-207.

Sabaté I., 2016 «The Spanish mortgage crisis and the re-emergence of moral economies in uncertain times», in History and Anthropology, 27 (1): 107-120.

Sachs W., 2010 «Preface to the new edition», in The Development Dictionary: A guide to Knowledge as Power, $2^{\text {nd }}$ edition, Zed Books, London: vi-xiv.

Sage C., 2012 Environment and Food, Routledge, London.

Sartorio A., 2008 Il mercante di utopie, Sperling \& Kupfer, Milano.

Sassatelli R. et al., 2015 Fronteggiare la crisi. Come cambia lo stile di vita del ceto medio, il Mulino, Bologna.

Sonnino R., Griggs-Trevarthen C., 2013 «A resilient social economy? Insights from the community food sector in the UK», in Entrepreneurship \& Regional Development, 25 (3-4): 272-292.

Stevenson R., 2002 «Dixons in £230m UniEuro takeover». https://www.independent.co.uk/news/ business/news/dixons-in-pound230m-unieuro-takeover-613617.html (ultimo accesso 10/03/17).

Tassan M., 2017 «Cibo "naturale" e food activism. Il consumo critico in due Gruppi di Acquisto Solidale nell'area Milanese», in Archivio Antropologico Mediterraneo, XX, n. 19 (1): 23-31.

Teughels N., Scholliers P. (eds), 2015 A Taste of Progress: Food at International and World Exhibitions in the Nineteenth and Twentieth Centuries, London, Routledge.

Theodossopoulos D., 2013 «Infuriated with the infuriated? Blaming tactics and discontent about the Greek financial crisis», in Current Anthropology, 54 (2): 200-221.

Varoufakis Y., 2016 I deboli sono destinati a soffrire? L'Europa, l'austerità e la minaccia alla stabilità globale, La nave di Teseo, Milano.

Vournelis L., 2013 «Paying the check, eating the money: foodbased challenges to neoliberalism in Greece», in Food, Culture \& Society, 16 (3): 354-359.

Watts D. et al., 2005 «Making reconnections in agro-food geography: alternative systems of food provision», in Progress in Human Geography, 29 (1): 22-40.

Wiskerke J. S. C., 2009 «On places lost and places regained: reflections on the alternative food geography and sustainable regional development», in International Planning Studies, 14 (4): 369-387.

\section{NOTE}

1. Quando parlo di "merce" intendo il processo di trasformazione di qualcosa in una merce, non un oggetto specifico. Merci specifiche possono assumere ruoli diversi in contesti diversi, per questo dobbiamo pensare in termini di processi di mercificazione anziché di oggetti che rimangono, sempre e comunque, merci.

2. Come nota Wiskerke, però, "molto spesso [sono] i cibi alternativi a essere prodotti, trasformati, distribuiti e consumati in molte di queste reti alternative" (2009: 378, traduzione e corsivi miei), complicando ulteriormente la possibilità di distinguere tra le due interpretazioni. 
3. Per i molti volti di questa riscoperta (dei quali non tutti possono essere considerati positivi) vedi: Crouch et al. (2016); Della Porta (2015); Lekakis (2017); Narotzky (2016); Sabaté (2016); Theodossopoulos (2013).

4. Nel 2015 la raccolta dati è stata condotta dall'autore sotto la supervisione della Professoressa Francesca Forno, presso l'Osservatorio CORES (Consumi, reti e pratiche di economie sostenibili), Dipartimento di Scienze Umane e Sociali, Università di Bergamo, con finanziamento Progetto ITALY $Y^{\oplus}$ - Azione: Giovani in Ricerca 2014. La stesura dell'articolo ha ricevuto un contributo dal Settimo Programma Quadro dell'Unione Europea, finanziamento Marie Skłodowska-Curie N. 609402-2020 researchers: Train to Move.

5. Vedi Teughels, Scholliers (2015) per un'analisi storica del ruolo del cibo nelle Esposizioni Universali.

6. Per una lista completa dei partner vedi http://www.expo2015.org/partner/ (ultimo accesso 14/02/17).

7. Tutti i nomi di persona usati nell'articolo sono pseudonimi.

8. Il fatto di non avere ripreso a produrre parti per automobili non sconfessa, per gli exlavoratori, il loro progetto di "impresa recuperata". Ai loro occhi, la continuità è dettata dal fatto che un gruppo di essi ha mantenuto vivo il sito della fabbrica, pur destinandolo ad altri usi. Dopo lunghe discussioni interne, il gruppo ha deciso che i grandi spazi di cui disponeva la fabbrica ne costituivano il principale punto di forza e potevano essere trasformati in qualcosa che assomigliasse a un incrocio tra un centro polivalente per la comunità locale e uno spazio di cowork. Il capannone più vicino alla strada è stato trasformato in uno spazio utilizzato da gruppi locali per concerti, riunioni, proiezioni di film, spettacoli teatrali e corsi. Esso ospita anche un mercato dove artigiani locali vendono prodotti che vanno dai mobili agli abiti fatti in casa. Ci sono poi una tappezzeria e una carpenteria, un "laboratorio" che ricondiziona apparecchi elettronici e uno che ripara i bancali (le strutture in legno usate per trasportare merci) per imprese locali. Il secondo capannone è stato riadattato in parcheggio per camper, mentre il terzo è occupato da un'azienda che ricicla carta e plastica. Infine, il quarto capannone è diventato un deposito usato perlopiù da persone che non si possono permettere garage o magazzini commerciali. Altri spazi sono affittati a bande musicali e appassionati di modellismo. Ci sono anche un bar e una cucina che serve pasti a chi si reca in fabbrica per partecipare alle sue attività culturali o per utilizzare i laboratori.

9. Le motivazioni di questa interpretazione sono complesse e possono essere qui solo accennate. In sostanza, le norme europee sono considerate troppo costose e complesse per le aziende famigliari (spesso micro-aziende), creando così un impedimento alla loro partecipazione al mercato degli alimenti a valore aggiunto. Esse sono inoltre considerate per lo più inutili, soprattutto dal punto di vista igienico-sanitario, poiché si ritiene che gli scandali alimentari siano provocati dalle produzioni di massa e non da quelle casalinghe.

\section{RIASSUNTI}

L'articolo si chiede in che modo sono cambiate le reti alternative del cibo italiane (alternative food networks) a partire dalla crisi economica globale del 2008. Esso analizza il fenomeno in questione tramite il ricorso a due letterature scientifiche di riferimento, quella dell'antropologia del valore, declinata sugli studi del cibo, e quella dei regimi alimentari (food regimes). Dopo avere discusso la letteratura alla luce della domanda di fondo, l'articolo sviluppa quattro ipotesi di lavoro per 
tentare di rispondervi. Le ipotesi identificate sono: 1) generazione, 2) rafforzamento, 3) ostruzione e 4) irrilevanza. Nella seconda metà dell'articolo, si prende in esame un caso specifico di rete alternativa del cibo, Fuori Mercato, come possibile illustrazione della prima ipotesi, quella della generazione.

This article asks how Italian alternative food networks have changed after the global economic crisis of 2008. The article analyzes the phenomenon in question through the use of two bodies of literature, the anthropology of value (deployed in the direction of food studies) and food regimes theory. After discussing the literature in reference to the central question, the article develops four hypotheses to try to answer it. These are: 1) generation, 2) strengthening, 3) obstruction and 4) irrelevance. In the second half of the article, a specific example of alternative food network called Fuori Mercato ('outside the market') is examined as a possible illustration of the first hypothesis, that of generation.

\section{INDICE}

Keywords : food, social movements, austerity, commodities, organic agriculture

Parole chiave : cibo, movimenti sociali, austerità, merci, agricoltura biologica

\section{AUTORE}

\section{GIOVANNI ORLANDO}

Dipartimento di Culture, Politiche e Società, Università di Torino giovanni.orlando[at]unito.it 